\title{
Targeting adipose tissue angiogenesis to enhance insulin sensitivity
}

\author{
L. Mannerås-Holm • A. Krook
}

Received: 21 June 2012 / Accepted: 27 June 2012 / Published online: 29 July 2012

(C) The Author(s) 2012. This article is published with open access at Springerlink.com

Keywords Adipose tissue · Angiogenesis · Insulin resistance · Rosiglitazone $\cdot$ Thiazolidinediones $\cdot$ Vascularisation

$\begin{array}{ll}\text { Abbreviations } \\ \text { PPAR } \gamma & \text { Peroxisome proliferator-activated receptor } \gamma \\ \text { TZD } & \text { Thiazolidinediones } \\ \text { VEGFA } & \text { Vascular endothelial factor A }\end{array}$

Adipose tissue plays an essential role in metabolic homeostasis. Abnormal expansion of (obesity) and markedly reduced (lipodystrophy) adipose tissue mass represent the extreme opposite ends of the adiposity spectrum. Intriguingly, both lead to metabolic dysfunction including insulin resistance and type 2 diabetes. Furthermore, adipose tissue is today recognised as an important endocrine organ and dysregulated secretion of adipokines may lead to profound changes in insulin sensitivity.

In the current issue of Diabetologia, Gealekman et al present evidence that targeting adipose tissue angiogenesis may promote insulin sensitivity (Fig. 1a) [1]. The primary function of adipose tissue is to store energy in the form of lipids in situations of energy excess, and release it when energy is needed. Problems occur when the lipid storage capacity of adipose tissue is exceeded, which leads to ectopic deposition of lipids in insulin-sensitive tissues, including liver and muscle. The central role of adipose tissue in whole-body

L. Mannerås-Holm

Department of Molecular Medicine and Surgery,

Karolinska Institutet,

Stockholm, Sweden

A. $\operatorname{Krook}(\square)$

Department of Physiology and Pharmacology,

Karolinska Institutet,

von Eulers Road 4a,

17177 Stockholm, Sweden

e-mail: Anna.Krook@ki.se energy homeostasis makes it an attractive potential therapeutic target. However, because it is involved in a vast array of physiological processes throughout the body, adipose tissue is also a challenging target.

Unlike most other adult tissues, adipose tissue displays plasticity and has an enormous capacity to expand and regress throughout our lifetime. Expansion of adipose tissue requires coordinated cellular processes, including an increase in volume and/or number of fat cells and alterations in the surrounding matrix and vascularisation. Indeed, recent evidence from mice with a targeted deletion of $\mathrm{Fgfl}$ indicates that a failure of the ability of adipose tissue to expand and contract in response to metabolic demands leads to metabolic disease [2]. Adipose tissue angiogenesis (i.e. growth of new blood vessels) ensures delivery of oxygen and nutrients, growth factors, hormones, stem cells and immune cells, as well as removal of waste products and transportation of fatty acids and adipokines into the circulation [3]. Thus, adipogenesis and angiogenesis are two closely related processes. A low angiogenic capacity could decrease adipogenic potential and consequently lead to adipocyte hypertrophy, which, in turn, is closely related to insulin resistance. Adipocytes themselves produce several angiogenic factors, including leptin, vascular endothelial growth factor A (VEGFA) and TNF- $\alpha$. Conversely, angiogenic factors such as VEGFA can modulate adipocyte differentiation [3].

When adipose tissue expansion exceeds compensatory vascular supply, hypoxia may develop. Local adipose tissue hypoxia has been proposed as a mechanism leading to dysregulation of adipose tissue function in the context of obesity and insulin resistance [3]. Thus, improving angiogenesis may be a novel approach to tackle adipose tissue hypoxia and hence promote insulin sensitivity. However, in contrast to most rodent models of obesity implicating adipose tissue hypoxia as a factor inducing adipose tissue dysfunction, a recent report indicates that in obese insulin-resistant 
individuals there is adipose tissue hyperoxia. The hyperoxia is present despite lower adipose tissue blood flow, possibly due to lower oxygen consumption by obese adipose tissue [4].

Anti-angiogenic drugs are being used as first-line treatment for various cancers. Like tumours, adipose tissue depends on proper vascularisation to grow, and so inhibition of adipose tissue angiogenesis may inhibit adipose tissue expansion and thus the development of obesity. Indeed, systemic administration of anti-angiogenic agents in different mouse obesity models has shown promising results, including dosedependent weight reduction and loss of fat mass (Fig. 1b) [3, 5]. This was, however, partly explained by reduced food intake [3]. Furthermore, inhibition of adipose tissue expansion achieved by reducing adipose tissue vascularisation might lead to lipotoxicity because of increased lipolysis and/or ectopic fat deposition, particularly if energy intake is not simultaneously decreased. Furthermore, inhibition of angiogenesis may have adverse effects in metabolically active brown adipose tissue, which may, in turn, lower energy expenditure.

Impaired adipose tissue angiogenesis could potentially limit adipogenesis and thereby contribute to metabolic dysfunction by promoting ectopic lipid accumulation [6]. Thus, the converse approach, i.e. enhancing adipose tissue angiogenesis, preferably in the subcutaneous depot, should promote fat storage in the appropriate tissue and subsequently protect against metabolic dysfunction. One of the few groups of therapeutic agents to directly target adipose tissue is the thiazolidinediones (TZDs, e.g. rosiglitazone), a class of peroxisome proliferator-activated receptor $\gamma$ (PPAR $\gamma$ ) agonists. These drugs improve insulin sensitivity by promoting adipogenesis and fatty acid uptake in adipose tissue and thereby sequester lipids away from non-fat tissues. Consequently, metabolic function is improved despite increased fat mass by

a

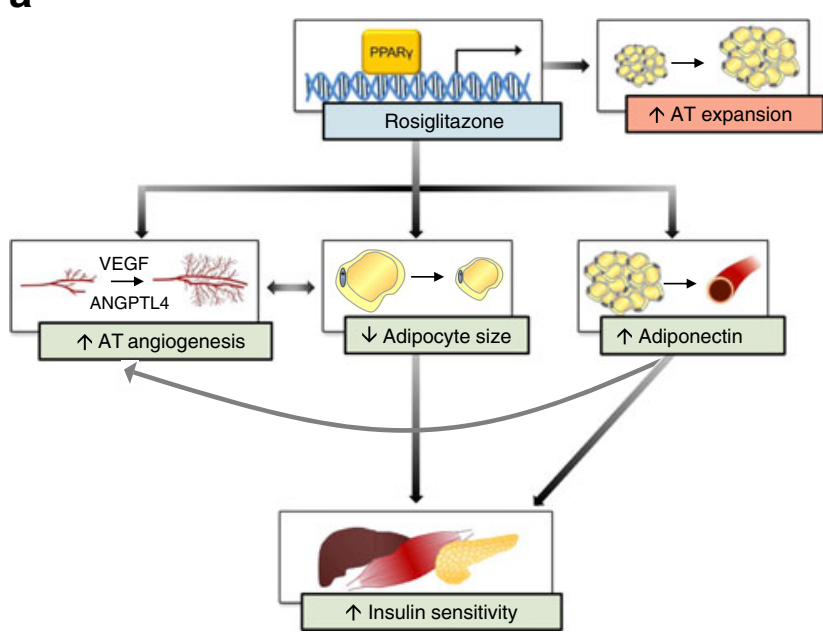

Fig. 1 Proposed mechanisms whereby enhancing (a) or inhibiting (b) adipose tissue (AT) angiogenesis leads to increased insulin sensitivity. (a) Outline of approached presented in [1, 8]. Green boxes denote an increase in the amount of subcutaneous fat and a decrease in the amount of visceral fat [7]. In the current issue of Diabetologia, Gealekman et al report that short-term treatment (6 weeks) with rosiglitazone in overweight and obese individuals results in alterations in adipose tissue architecture in terms of increased formation of small adipocytes and increased capillary density (Fig. 1a) [1]. The authors suggest that the improved adipose tissue vascularisation may in part mediate the therapeutic actions of rosiglitazone and that adipose tissue vascularisation can directly be modulated by PPAR $\gamma$ activation. The same research group has previously reported a correlation between angiogenic capacity in subcutaneous adipose tissue and insulin sensitivity assessed by HOMA of insulin resistance (HOMA-IR) [6]. Intriguingly, in the present study, the improved TZD-induced adipose tissue vascularisation was not accompanied by an improvement in HOMA-IR. This might partly be explained by the fact that only volunteers with normal glucose tolerance were included in the study.

TZDs are known to alter the production of adipokines (for example, increased adiponectin and reduced TNF- $\alpha$ and leptin). In accordance with previous studies, Gealekman et al observed increased circulating adiponectin levels in response to rosiglitazone treatment [1]. Adiponectin levels were positively correlated with capillary growth rate. Adiponectin has been shown to have both anti- and pro-angiogenic activity, making it difficult to interpret its role in adipose tissue angiogenesis and involvement in rosiglitazone-induced vascularisation.

The VEGF system is considered to be responsible for the majority of angiogenic activity in adipose tissue [3]. Overexpression of Vegfa specifically in adipose tissue of dietinduced obese mice results in enhanced vascularisation and browning of adipose tissue accompanied with improved insulin sensitivity and higher energy expenditure [5]. Consistent b

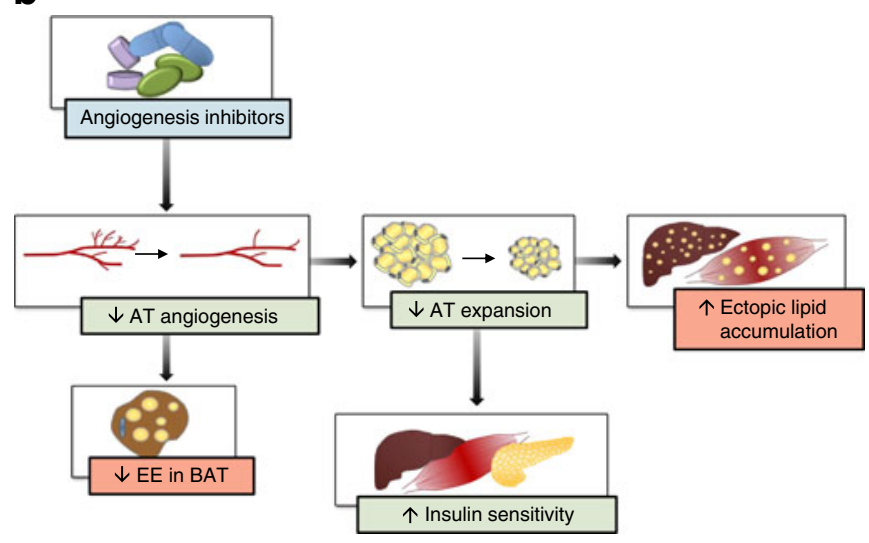

inferred favourable effects, while red boxes indicate possible detrimental side-effects. AT, adipose tissue; BAT, brown adipose tissue; EE, energy expenditure 
with the present study, Gealekman et al previously reported smaller fat cells and increased capillary density following rosiglitazone treatment in obese and diabetic rodents [8]. The angiogenic effect of rosiglitazone in rodents was partly attributed to increased Vegfa expression and angiopoietinrelated protein 4 production [8]. Unfortunately, the expression of angiogenic factors was not evaluated in the present study. In contrast to these studies, rosiglitazone has been shown to have anti-angiogenic effects leading to suppression of primary tumour growth and metastasis [9]. Notably, rosiglitazone has been withdrawn from the market owing to an increased risk of cardiovascular events, which makes the reported results challenging to translate to the clinical situation.

Maintaining adequate function in expanding adipose tissue is dependent on angiogenesis, and adipose tissue mass can thus be regulated through the vasculature. TZDs improve insulin sensitivity despite paradoxically increasing fat mass. The paper in the current issue of Diabetologia [1], suggests that one mechanism by which TZDs improve insulin resistance is by stimulating angiogenesis, thereby promoting adipogenesis in subcutaneous adipose tissue (Fig. 1a).

The relationship between adipose tissue vascularisation and obesity in humans remains to be established. Some reports indicate lower vascular density with increasing BMI $[4,6,10]$, while others note the opposite [11]. This discrepancy may be explained at least in part by heterogeneity in the study populations, such as different stages of obesity and insulin sensitivity. Furthermore, Gealekman et al have previously examined angiogenic capacity and capillary density in different adipose tissue depots, and report that subcutanous adipose tissue has a higher capillary density than visceral adipose tissue [6].

Due to the paucity of preclinical and clinical studies, it is difficult to draw firm conclusions as to whether angiogenic mediators may be a fruitful approach to regulate adipose tissue function and mass (Fig. 1). Furthermore, current reports demonstrate improved metabolic function in response to either enhancing or inhibiting adipose tissue angiogenesis $[3,5,8]$. This paradox highlights the complexity of interfering with adipose tissue angiogenesis (as well as possible off-target effects), and it is uncertain whether stimulation or inhibition of adipose tissue angiogenesis is preferable (Fig. 1). While some obesity-related complications may benefit from inhibition of adipose tissue angiogenesis (e.g. type 2 diabetes, inflammation and cancer), others may benefit from stimulation of adipose tissue angiogenesis (e.g. hypertension and cardiovascular diseases) [3]. Tissues that are highly metabolically active, such as brown adipose tissue, may derive an advantage from increased angiogenesis by increasing energy expenditure. If and when we see the advent of angiogenic modulators as a treatment of obesity and related metabolic disorders, they clearly need to be tissue-specific. Since adipose tissue is one of the largest organs in the body, high doses of angiogenic modulators may be required to target this tissue, and consequently the risk of side effects is high. Nonetheless, modulation of adipose angiogenesis remains an intriguing approach to improve insulin sensitivity in the context of metabolic disease.

Funding L. Mannerås-Holm is supported by a post-doctoral training fellowship from the Swedish research council (2010-6739).

Duality of interest The authors declare that there is no duality of interest associated with this manuscript.

Contribution statement $\mathrm{LMH}$ and $\mathrm{AK}$ were responsible for the conception, design and drafting of the manuscript, and approved the final version for publication.

Open Access This article is distributed under the terms of the Creative Commons Attribution Noncommercial License which permits any noncommercial use, distribution, and reproduction in any medium, provided the original author(s) and the source are credited.

\section{References}

1. Gealekman O, Guseva N, Gurav K, et al (2012) Effect of rosiglitazone on capillary density and angiogenesis in adipose tissue of normoglycemic humans in a randomized controlled trial. Diabetologia

2. Jonker JW, Suh JM, Atkins AR et al (2012) A PPAR $\gamma$-FGF1 axis is required for adaptive adipose remodelling and metabolic homeostasis. Nature 485:391-394

3. Cao Y (2010) Adipose tissue angiogenesis as a therapeutic target for obesity and metabolic diseases. Nat Rev Drug Discov 9:107-115

4. Goossens GH, Bizzarri A, Venteclef $N$ et al (2011) Increased adipose tissue oxygen tension in obese compared with lean men is accompanied by insulin resistance, impaired adipose tissue capillarization, and inflammation. Circulation 124:67-76

5. Sun K, Wernstedt Asterholm I, Kusminski CM et al (2012) Dichotomous effects of VEGF-A on adipose tissue dysfunction. Proc Natl Acad Sci U S A 109:5874-5879

6. Gealekman O, Guseva N, Hartigan C et al (2011) Depot-specific differences and insufficient subcutaneous adipose tissue angiogenesis in human obesity. Circulation 123:186-194

7. Mori Y, Murakawa Y, Okada K et al (1999) Effect of troglitazone on body fat distribution in type 2 diabetic patients. Diabetes Care 22:908-912

8. Gealekman O, Burkart A, Chouinard M, Nicoloro SM, Straubhaar J, Corvera S (2008) Enhanced angiogenesis in obesity and in response to PPAR $\gamma$ activators through adipocyte VEGF and ANGPTL4 production. Am J Physiol Endocrinol Metab 295:E1056-E1064

9. Panigrahy D, Singer S, Shen LQ et al (2002) PPAR $\gamma$ ligands inhibit primary tumor growth and metastasis by inhibiting angiogenesis. J Clin Invest 110:923-932

10. Spencer M, Unal R, Zhu B et al (2011) Adipose tissue extracellular matrix and vascular abnormalities in obesity and insulin resistance. J Clin Endocrinol Metab 96:E1990-E1998

11. Lemoine AY, Ledoux S, Queguiner I et al (2012) Link between adipose tissue angiogenesis and fat accumulation in severely obese subjects. J Clin Endocrinol Metab 97:E775-E780 